Research Paper

\title{
Switch of the ovarian cancer cell to a calcifying phenotype in the calcification of ovarian cancer
}

\author{
Jirui Wen, ${ }^{1,2^{*}}$ Zhiwei Zhao, ${ }^{2 *}$ Liwei Huang, ${ }^{3}$ Liang Li, ${ }^{2}$ Jiman Li, ${ }^{4}$ Ye Zeng, ${ }^{2}$ Jiang Wu, ${ }^{2}$ Yali Miao ${ }^{\bowtie}$ \\ 1. Department of Obstetrics and Gynecology, Key Laboratory of Birth Defects and Related Diseases of Women and Children of MOE, West China Second \\ University Hospital, Sichuan University, Chengdu, P.R. China \\ 2. West China School of Basic Medical Sciences \& Forensic Medicine, Sichuan University, Chengdu, China \\ 3. West China School of Stomatology Medicine, Sichuan University, Chengdu, China \\ 4. Pathology Department, Sichuan Cancer Hospital, Chengdu, China \\ *The first two authors made an equal contribution to the paper.
}

$\triangle$ Corresponding authors: Prof. Yali Miao, PhD, Department of Obstetrics and Gynecology, Key Laboratory of Birth Defects and Related Diseases of Women and Children of MOE, West China Second University Hospital, Sichuan University, No17, 3rd section, Renmin Nanlu Road, Road, Chengdu 610041, Sichuan, China. Tel: +8618280303652; E-mail: miaoyali2006@163.com and Prof. Jiang Wu, MD, PhD, West China School of Basic Medical Sciences \& Forensic Medicine, Sichuan University, No17, 3rd section, Renmin Nanlu Road, Road, Chengdu 610041, Sichuan, China. E-mail: aa806678836@163.com

(c) Ivyspring International Publisher. This is an open access article distributed under the terms of the Creative Commons Attribution (CC BY-NC) license (https://creativecommons.org/licenses/by-nc/4.0/). See http://ivyspring.com/terms for full terms and conditions.

Received: 2017.09.21; Accepted: 2018.01.28; Published: 2018.02.28

\begin{abstract}
Objective: The main aim of this study was to study swith of the ovarian cancer cell to a calcifying phenotype in the formation of calcification in ovarian cancer, and to offer some help for ovarian cancer's diagnosis and differentiation therapy.

Methods: The mineralization of ovarian cancer cell lines SKOV3 was induced via calcification medium for $21 \mathrm{~d}$ in vitro. Alizarin red staining, von kossa staining, calcein fluorescence staining and ALP activity detection were used to identify mineralization in calcification model of ovarian cancer. Also, the changes of ultrastructure and the mineralization biomarkers after the induction of calcification medium were investigated by transmission electron microscopy and western blot, respectively. The SKOV3 cells migration behavior after the induction of calcification medium was evaluated by using transwell assay and scratch wound. Finally, mineralization biomarkers were verified in 40 cases of calcified ovarian cancer specimens and matched 40 non-calcified ovarian cancer tissues.

Results: Classical calcium salt detection methods confirmed that the culture of SKOV3 cells in calcification medium was an appropriate ovarian cancer calcification model in vitro. Transmission electron microscopy and western blot revealed respectively the presence of cells with morphological characteristics of osteoblasts and the upregulation of mineralization biomarkers expression in treatment group. Transwell assay and scratch wound showed the decreased SKOV3 cell migration in treatment group. In specimens, the calcification occurred predominantly in well-differentiated carcinomas and the expression of the BMP2 and OPN elevated in calcified group. Conclusion: Our study showed that the switch of the ovarian cancer cell to a calcifying phenotype in the formation of calcification in ovarian cancer. The calcified phenotypic transformation may inform the new prospective in ovarian cancer therapy.
\end{abstract}

Key words: ovarian cancer; calcification; migration; phenotypic transformation; differentiation therapy

\section{Introduction}

Calcification represents one of the common imaging features of ovarian cancer. It occurs in $8 \%$ ovarian cancer, mainly in serous ovarian cancer[3]. For serous ovarian cancer, calcification mainly exists as psammoma body, which is arranged as concentric circles[4]. As a specific pathologic sign, psammoma body has been used as one of the pathologic evidences to diagnose ovarian serous papillary carcinoma[4]. 
Although calcification in ovarian cancer has an important clinical significance, it remains largely unexplored and poorly understood.

Currently, one of the unclear things about calcification in ovarian cancer is the mechanism by which calcification forms. The formation of calcification in ovarian cancer might be similar to the ectopic calcification. Ectopic calcification refers to calcium deposition which happens outside osseous tissue and defers from normal calcification. Ectopic calcification is divided into dystrophic calcification and metastatic calcification. Dystrophic calcification is caused by tissue necrosis while metastatic calcification is induced by the metabolic disorder of calcium and phosphorus. Supporters of the former opinion consider that calcification in ovarian cancer is caused by the calcium deposition on necrotic tissue[5]. However, supporters of the latter opinion believe the formation of calcification is not related to necrosis[6]. They believe that the formation of calcifications in ovarian cancer is similar to the metastatic calcification, as the result of the disorder of the local calcium metabolism. The disorder may be caused by the increased secretion of ovarian cancer cells. Das and colleagues believe that the formation of calcifications may be linked to the secretion of collagen from the papillary serous ovarian cancer[4]. Silva and colleagues find that calcification is predominant in the matrix and can be induced by multiple hormones, which suggested that calcification may be secondary to the secretion of the cancer cells, but not to necrosis[7]. So why ovarian cancer cells have a high level of secretion? It may be related to the cell phenotype transformation according to the recent studies. In vascular calcification, vascular smooth muscle cells switch from a contractile phenotype to a calcifying phenotype and express bone-related transcription factors and bone matrix proteins to play an essential role in the process of vascular calcification [8]. In dysplasia progressive osteitis, local inflammatory signals and changes in the microenvironment of the tissue can mediate the differentiation of the mesenchymal stem cells to cartilage cells and osteoblasts. Similar findings also have been found in the tumor. Manuel Scimeca found that in breast cancer, the deposition of local calcification is due to the secretion of some calcifying cells[9].

According to the findings above, we can hypothesize that ovarian cancer cells switch to calcifying cells in certain conditions and lead to the local calcium deposition. Therefore, the main aim of this study was to investigate the formation of calcification in ovarian cancer, and to offer some help for ovarian cancer's diagnosis and therapy.

\section{Material and Methods}

\section{Cell culture}

Human ovarian cancer cells SKOV3 and human ovarian epithelial cells HOSEpiC were obtained from Nanjing KeyGen Biotech.Inc. Cells were cultured in Alfa modified Eagle's medium ( $\alpha$-MEM, Hyclone, USA) supplemented with $10 \%$ fetal bovine serum (FBS, Israel) and $1 \%$ penicillin-streptomycin (Hyclone, USA) at $37{ }^{\circ} \mathrm{C}$ with $5 \% \quad \mathrm{CO}_{2}$ saturation, and the medium was changed every 3 days. The cells used for experiments were after four passages.

\section{Assessment of mineralization in vitro}

SKOV3 or HOSEpiC cells were seeded into six-well culture plates (day 0 ) at $1.5 \times 10^{3}$ cells per well. Then the cells were treated in culture medium or calcification medium. Culture medium was a-MEM supplemented with $10 \%$ fetal bovine serum, $50 \mathrm{U} / \mathrm{ml}$ penicillin, $50 \mathrm{mg} / \mathrm{ml}$ streptomyocin. Calcification medium was culture medium supplemented with an osteogenic cocktail $(50 \mu \mathrm{g} / \mathrm{ml}$ vitamin C, $10 \mathrm{mM}$ beta glycerin sodium phosphate $+10^{-7} \mathrm{M}$ dexamethasone). Cells were cultured for up to 21 days in a humidified incubator at $37^{\circ} \mathrm{C}$ and $5 \% \mathrm{CO}_{2}$. The SKOV3 cells cultured with calcification medium for $21 \mathrm{~d}$ were used as treatment group, and the SKOV3 cells cultured with culture medium for 21d were used as control group.

Mineralization was assessed using alizarin red S staining (Shanghai Chemical Reagent Factory), von Kossa staining (Beijing Leagene Biotechnology Co., Ltd.), calcein fluorescence staining (Shanghai Shifeng Biotechnology Co., Ltd.) and ALP activity detection (Nanjing Institute Biological Engineering) at the 21 day, as described below.

\section{Alizarin red staining}

After the medium was removed, cells were rinsed with PBS and fixed with 95\% ethanol for 15 min. Then, the cells were rinsed twice with PBS and stained with alizarin red S (2\%, pH 4.4) for $5 \mathrm{~min}$. Finally, the cells were washed with PBS three times, and images were collected.

\section{Von Kossa staining}

After the medium was removed, cells were rinsed with PBS and fixed with 95\% ethanol for 15 min. Then, the cells were rinsed twice with PBS and stained with silver nitrate (5\%) for 1 h under an electric lamp. Next, sodium thiosulphate $(5 \%)$ was applied for 2 min. Finally, the cells were washed with PBS, and images were collected.

\section{Calcein fluorescence staining}

Cells was cultured in medium containing 5 
$\mathrm{ug} / \mathrm{ml}$ calcein (day 18) for $3 \mathrm{~d}$. After the medium was removed, SKOV3 cells were rinsed with PBS and fixed with $95 \%$ ethanol for $15 \mathrm{~min}$. Then fluorescent images were collected by using an inverted epifluorescence microscope (IX70; Olympus.Co., Tokyo, Japan)

\section{ALP activity detection}

Cell culture medium was used for the ALP assay. Samples $(50 \mathrm{ml})$ and p-nitrophenyl phosphate $(100 \mathrm{ml})$ were incubated at $37^{\circ} \mathrm{C}$ for $30 \mathrm{~min}$ and absorbance was measured at $520 \mathrm{~nm}$.

\section{Transmission electron microscopy (TEM)}

After the medium was removed, SKOV3 cells were rinsed with PBS and fixed with $3 \%$ glutaraldehyde precooling $4{ }^{\circ} \mathrm{C}$ overnight. Then, the cells were rinsed twice with PBS and fixed with $1 \%$ osmic acid $4{ }^{\circ} \mathrm{C}$ for $1 \mathrm{~h}$. After SKOV3 cells were rinsed again, the cells were dehydrated in ascending graded alcohols (30\% ethanol, $50 \%$ ethanol, $70 \%$ ethanol, $80 \%$ ethanol, $90 \%$ ethanol, $95 \%$ ethanol and $100 \%$ ethanol) for two $\times$ ten minute each. Then the cells were immersed in $70 \%, 80 \%, 90 \%, 95 \%, 100 \%$ acetonitrile solution fifteen minutes each. Then vacuum drying, spray coating of gold were performed. Finally, images were collected by scanning electron microscopy.

\section{Scratch wound}

At the $21 \mathrm{~d}$, the cells were synchronized at serum-free conditions overnight. Then a uniform scratch was performed using a plastic cell scrapper and the plates were rinsed with PBS and replenished with fresh serum-free medium. Images of the wounds were randomly chosen at $\mathrm{Oh}$ and photographed consecutively at $12 \mathrm{~h}$ and $24 \mathrm{~h}$. Also, the scratch area and the wound healing percentage was analysed consecutively at $0 \mathrm{~h}, 12 \mathrm{~h}$ and $24 \mathrm{~h}$. Three parallel experiments were done and data were presented as Mean \pm SD.

\section{Transwell assay}

After cultured for $21 \mathrm{~d}$, the SKOV3 cells with density of $2 \times 10^{6}$ cell $/ \mathrm{cm}^{2}$ were cultured in Transwell chamber $(8 \mu \mathrm{m}$ pore size; BD FalconTM, USA) for $24 \mathrm{~h}$ at $37^{\circ} \mathrm{C}$ and $5 \% \quad \mathrm{CO}_{2}$. After culturing, the inner chamber was cleaned, and the migrated cells on the bottom of the chamber was fixed and stained with $0.1 \%$ crystal violet. Each step was followed by washing with PBS for $5 \mathrm{~min}$ three times. The migrated cells were photographed at different fields of view using an inverted phase contrast microscope (CK2, Olympus, Japan). Also, the cell numbers of each group were calculated from images in triplicates.

\section{Western blot}

Cells cultured with calcification medium were rinsed twice times with PBS and disintegrated by 100 $\mu 1$ PIPA buffer (Beyotime, China) containing protease inhibitors and sodium orthovanadate (Beyotime, China). After 30 minutes, the samples were centrifuged with $10,000 \mathrm{rpm}$ at $4^{\circ} \mathrm{C}$ for $10 \mathrm{~min}$, and quantified by enhanced bicinchoninic acid (BCA) assay kit (Beyotime, China). Equal amounts of protein were electrophoresed in 10\% SDS-PAGE gels and transferred to polyvinylidene difluoride membranes (PVDF, GE Healthcare). Then the membranes were blocked with $5 \%$ milk in Tris-buffered saline with Tween 20 (TBST) at room temperature for 2 hours and incubated with anti-GAPDH (1:1000 dilution, sc-322 33, santa, USA), anti-Collagen I (1:1000 dilution, ab90 395, abcam, UK) , anti-Osterix (1:1000 dilution, ab225 52, abcam, UK), anti-OPN (1:1000 dilution, sc-21742, santa, USA), anti-BMP-2 (1:1000 dilution, sc-137087, santa, USA), anti- $\beta$ 2-microglobulin (1:1000 dilution, D8P1H,CST, USA) and anti-Runx2(1:1000 dilution, D1L7F, CST, USA) overnight at $4{ }^{\circ} \mathrm{C}$. Next, the membranes were washed three times for $5 \mathrm{~min}$ each and incubated with horseradish peroxidase (HRP) conjugating goat anti-mouse or goat-anti rabbit IgG (1:5000 dilution, Biosynthesis Biotechnology Co., LTD., China) for $2 \mathrm{~h}$ at $37^{\circ} \mathrm{C}$. Finally, the membranes were washed with TBST buffer for three times and visualized in Molecular Image ${ }^{\circledR}$ ChemiDocTM XRS+ system (Bio-Rad Inc., USA) with Image LabTM Software using enhanced chemiluminescence. The tests were performed three times and quantification was done and analyzed by Image J 1.44p software (National Institutes of Health, USA).

\section{Patients and tissue specimens}

All calcified ovarian cancer specimens $(n=40)$ and matched non-calcified ovarian cancer tissues $(n=40)$ were obtained from patients who underwent surgical treatment at the department of gynecology, Sichuan Provincial Cancer Hospital (Chengdu, China). Patients were diagnosed as serous ovarian cancer pathologically, and none of them received any other treatments prior to the harvest of the specimens. Written informed consents were obtained from all patients, and the study was approved by Sichuan University ethics committee.

\section{Ovarian cancer histology}

The ovarian cancer were sectioned, and the portions were fixed in $10 \%$ neutral buffered formalin, dehydrated, embedded in paraffin, sectioned at $5 \mu \mathrm{m}$, and stained with hematoxylin-eosin (H\&E).

\section{Immunohistochemistry}

The expression of Collagen I, Runx2, beta2microglobulin, BMP2, Osterix and OPN was 
investigated using immunohistochemisty (IHC) on formalin fixed, paraffin embedded sections by using the DAB kit (ZSGB-bIO, China). After pretreatment, sections were incubated with anti-Collagen I (ab903 95, abcam, UK) , anti-Osterix (ab22552, abcam, UK), anti-OPN (sc-21742, santa, USA), anti-BMP-2/ 4 (sc-13 7087, santa, USA), anti- $\beta$ 2-microglobulin (D8P1H, CST, USA)and anti-Runx2(D1L7F, CST, USA) .All the primary antibodies were at the 1:200 dilutions. Tissue sections were deparaffinized and rehydrated by passing the slides through xylene (3 washes, 10 minutes per wash) and graded alcohols (2 washes at $99.5 \%$ ethanol, 2 washes at $95 \%$ ethanol, 1 wash at $75 \%$ ethanol and 1 wash at $70 \%$ ethanol) for five minute each then placed and rinsed in water. The slides were incubated for $15 \mathrm{~min}$ with $0.6 \%$ hydrogen peroxide solution to block endogenous peroxidase activity. Slides were rinsed again and then underwent antigen retrieval. For heat-mediated antigen retrieval, slides were immersed in $0.1 \mathrm{M}$ citrate buffer $\mathrm{pH} 6.0$ and heated for $20 \mathrm{~min}$ at $95^{\circ} \mathrm{C}-99^{\circ} \mathrm{C}$. Slides naturally cooled to room temperature and then rinsed in phosphate buffered solution (PBS). Then attached one hundred microlitres of Protein Block was added to each slide for $30 \mathrm{~min}$ to prevent non-specific binding to any other reactive sites within the tissue. Then the slides were incubated with $40 \mu \mathrm{L}$ of the primary antibody at $4^{\circ} \mathrm{C}$ overnight. Following overnight incubation, slides were washed with $0.05 \%$ PBS 3 times per $5 \mathrm{~min}$. Then the slides were incubated with $40 \mu \mathrm{L}$ of the second antibody. After another wash, the slides were developed with the addition of $40 \mu \mathrm{L}$ 1:20-diaminobenzidine (DAB) solution followed by counterstaining by haematoxylin. Slides were then dehydrated in ascending graded alcohols (1 wash at $70 \%$ ethanol, 1 wash at $75 \%$ ethanol, 2 washes at $95 \%$ ethanol and 2 washes at $99.5 \%$; ethanol) for five minute each and cleared in 3 changes of xylene for three minute each. Finally, the sections were mounted using neutral gum and covered with a glass coverslip. Sections of appendix tissue with acute appendicitis were used as a positive control in each run of staining. For negative controls, slides were incubated with 40 $\mu \mathrm{L}$ antibody diluent instead of primary antibody.

\section{Assessment of expression}

The stained slides were then examined by light microscope to assess the expression of Collagen I, Runx2, beta2-microglobulin, BMP2, Osterix and OPN. The overall intensity of expression of Collagen I, Runx2, beta 2 - microglobulin, BMP2, Osterix and OPN in the serous tumor cells was scored as absent (0), weak (1) and strong (2). Score 0: Percentage of positive cells $<10 \%$; Score 1: Percentage of positive cells $>10 \%, \leq 50 \%$; Score 2: Percentage of positive cells
$>50 \%$.

\section{Statistical analysis}

In vitro, Statistical analysis was performed using GraphPad Prism 5 Software (La Jolla, CA, USA). P < 0.05 versus control group based on independentsamples t-test was considered significantly. In specimens, Statistical analysis was performed using the Chi-square test through SPSS (version 16.0, Chicago, IL, USA). Significant correlation was considered with $\mathrm{p}<0.05$.

\section{Results}

\section{In vitro calcification of SKOV3 ovarian cancer cells}

In order to study the molecular mechanisms of ovarian cancer calcification, it was necessary to firstly establish ovarian cancer calcification in vitro model and to determine whether the calcification of SKOV3 cells could be induced when cultured with the calcification medium. At 21 days, Alizarin Red staining (Figure 1A), von Kossa staining (Figure 1B) and calcein fluorescence staining (Figure 1C) confirmed the deposition of calcium in SKOV3 cells cultured with calcification medium respectively. In Alizarin Red staining, the strongest staining was observed in the SKOV3 cells cultured with calcification medium, rather than SKOV3 cells cultured with culture medium or HOSEpiC cells. In von kossa staining, positive staining was detected in the SKOV3 cells cultured with calcification medium. In calcein fluorescence staining, increased intensity and density of fluorescence staining were observed in the SKOV3 cells cultured with calcification medium. Additionally, the activity of ALP, a key indicator of calcification, was also significantly elevated $(\mathrm{P}<0.001$; Figure 1D). These results suggest that the culture of SKOV3 cells in calcification medium was an appropriate ovarian cancer calcification model in vitro.

\section{Ultrastructure characteristics of calcifying SKOV3 cells}

Transmission electron microscopy study revealed the presence of cells with ultrastructure characteristics of osteoblasts in treatment group (Figure 2). For SKOV3 cells cultured with calcification medium, compared with the control group, glycogen and fat particles loss seriously, metabolism slowed and intercellular junction was sparse (Figure 2B). The cytoplasm of calcifying SKOV3 cells were rich in vesicles containing electron-dense granules similar to the intracellular vesicle of the osteoblasts and these intracellular vesicles were secreted outside cells (Figure 2C). 

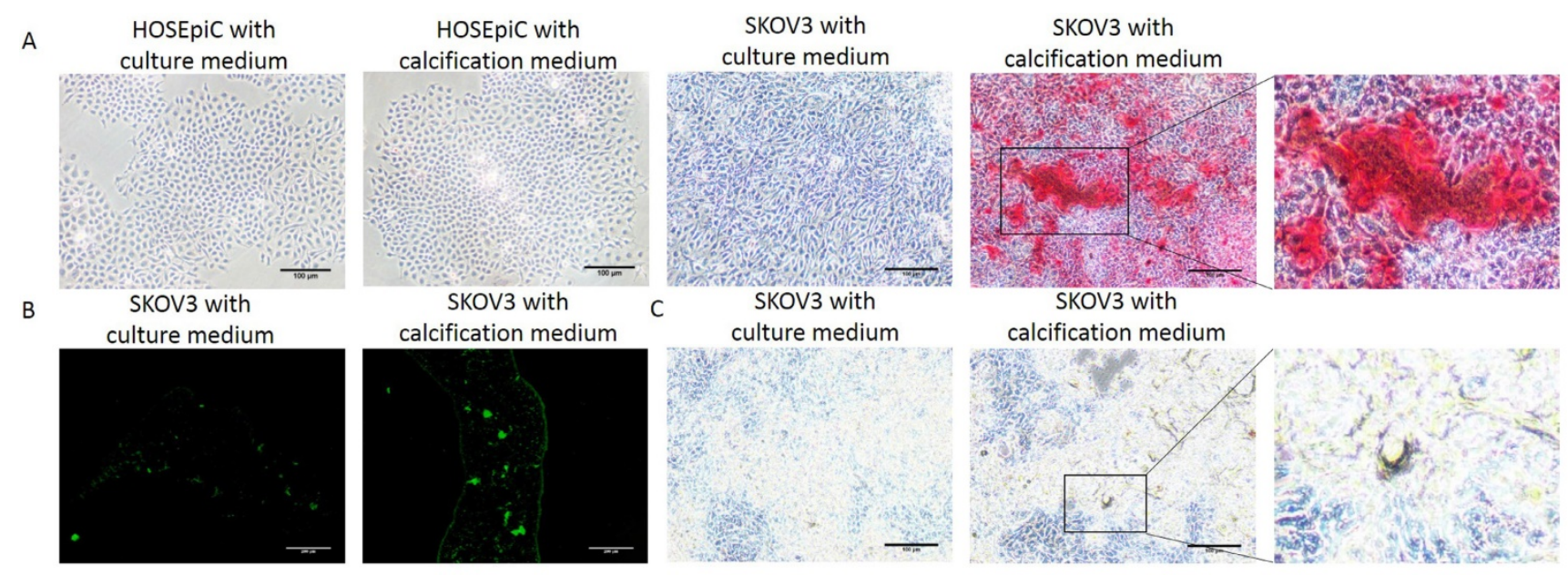

D

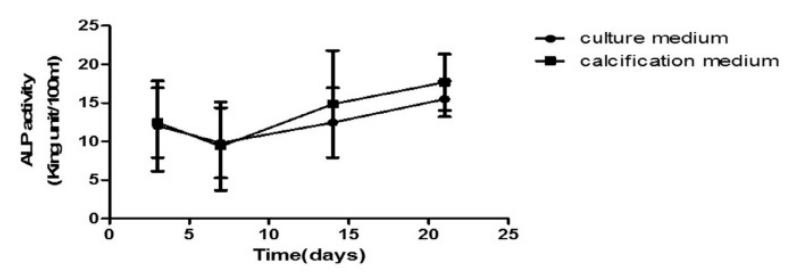

Figure 1. Detection of mineralization in vitro. (A) Alizarin Red staining, bar $=100 \mu \mathrm{m}$ (B) Calcein fluorescence staining, bar=200 $\mu \mathrm{m}$ (C) von Kossa staining,

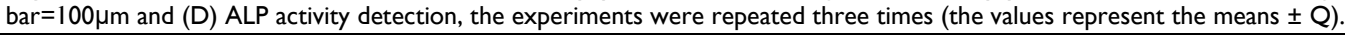

A
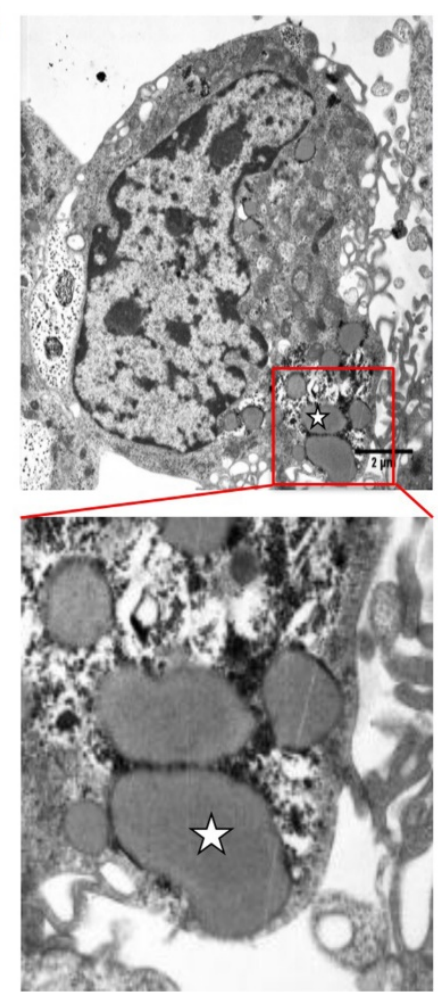

B

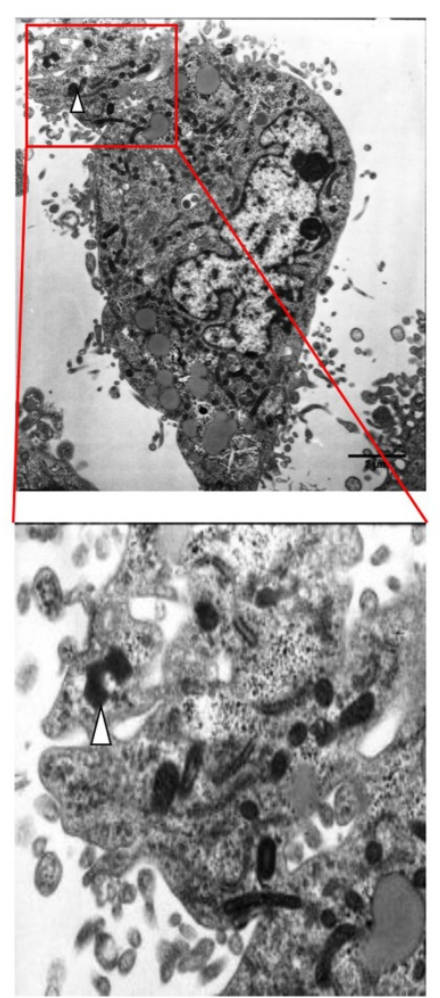

C
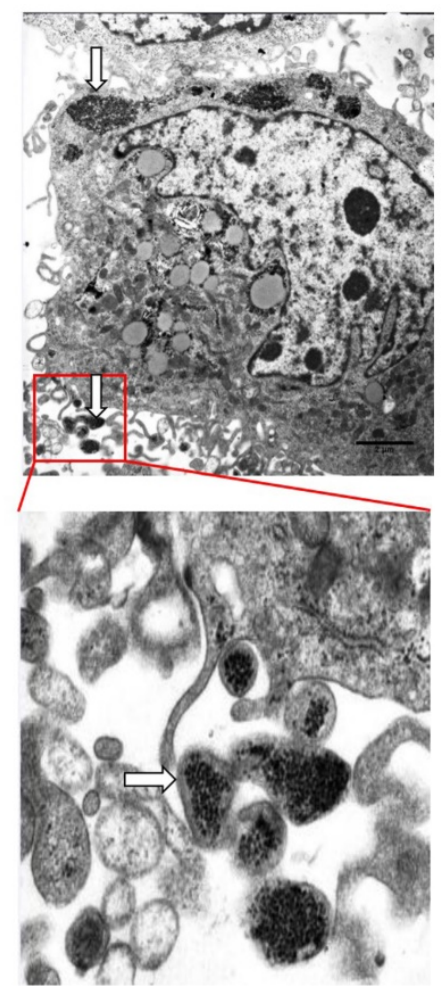

Figure 2. Electron micrograph obtained by TEM on control group and treatment group. (A) TEM on non-calcified SKOV3 cells in vitro. (B) (C) TEM on calcified SKOV3 cells in vitro. $\downarrow$, glycogen、fat particles. $\triangle$, secretion of cell degranulation, $\uparrow$, calcified vesicle, bar $=2 \mu \mathrm{m}$.

\section{Upregulation of mineralization biomarkers expression in calcifying SKOV3 cells}

In order to verify whether SKOV3 cells maintained a calcifying phenotype throughout the culture period, the expression of the mineralization biomarkers Collagen I, Runx2, $\beta 2$-microglobulin, BM P2, Osterix and OPN were confirmed by western blot (Figure 3A). Compared to control group, mineralizat- 
ion biomarkers Collagen I, Runx2, $\beta 2$-microglobulin, BMP2, Osterix and OPN upregulated in treatment group. Quantitative analysis revealed significant increase $(\mathrm{P}<0.05$; Figure $3 \mathrm{~B}, \mathrm{C}, \mathrm{D}, \mathrm{E}, \mathrm{F}, \mathrm{G})$ in the expression of Collagen I, Runx2, $\beta 2$-microglobulin, BMP2, Osterix in SKOV3 cells cultured with calcification medium.

\section{SKOV3 cell migration decreased after cultured with calcification medium for $21 \mathrm{~d}$}

The SKOV3 cell migration behavior was evaluated by using Transwell assay. It could be found that efficient SKOV3 cells migration through the filter of Transwell occurred in treatment group. By contrast, few SKOV3 cells across the Transwell membrane filter could be found in control groups (Figure 4A). Compared with the control groups, an increasing number of cells migrated in treatment groups (Figure 4B). Additionally, scratch wounds were made in monolayers of SKOV3 cells after cultured for 21d and a consistent conclusion with Transwell assay was revealed (Figure $4 \mathrm{C}$ ). At any time point, the results were found to be statistically significant by quantitative analysis of wound healing rate between control group and treatment group (Figure 4D). Collectively, the results suggest a decreased migration of calcifying SKOV3 cells.
A

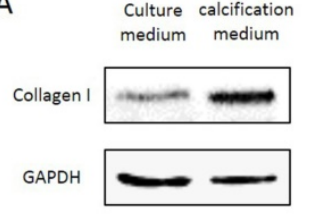

Runx2

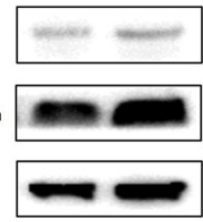

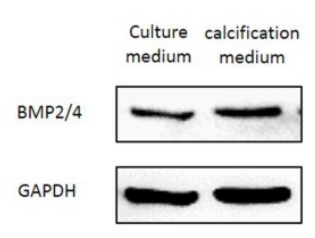

Osterix

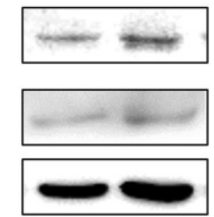

B

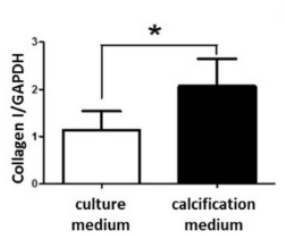

E

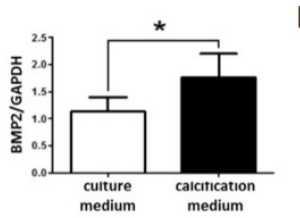

C

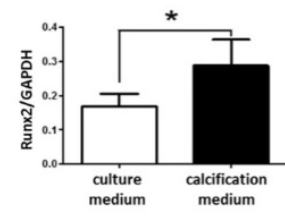

$\mathrm{F}$

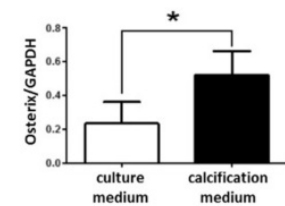

D

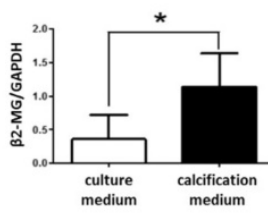

G

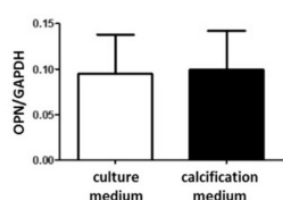

Figure 3. Expression of Collagen I, Runx2, $\beta 2$-microglobulin, BMP2, Osterix and OPN in control group and treatment group. (A) Western blotting analyses of Collagen I, Runx2, $\beta 2$-microglobulin, BMP2, Osterix and OPN protein levels in control group and treatment group. GAPDH served as a loading control. Statistical results for Collagen I, Runx2, B2-microglobulin, BMP2, Osterix and OPN protein levels in control group and treatment group are illustrated in (B), (C), (D), (E), (F) and (G), respectively. The experiments were repeated three times (the values represent the means $\pm S D, * P<0.05$ versus control group based on independent-samples t-test).

A
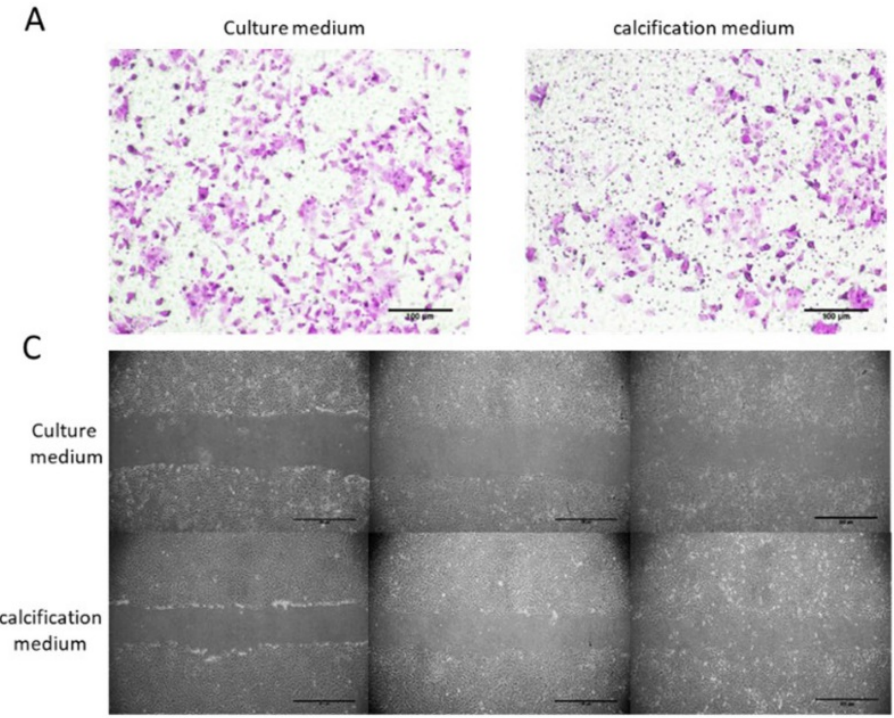

calcification medium

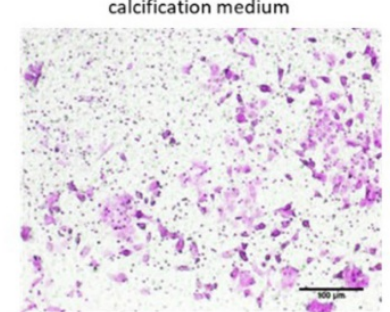

D

B
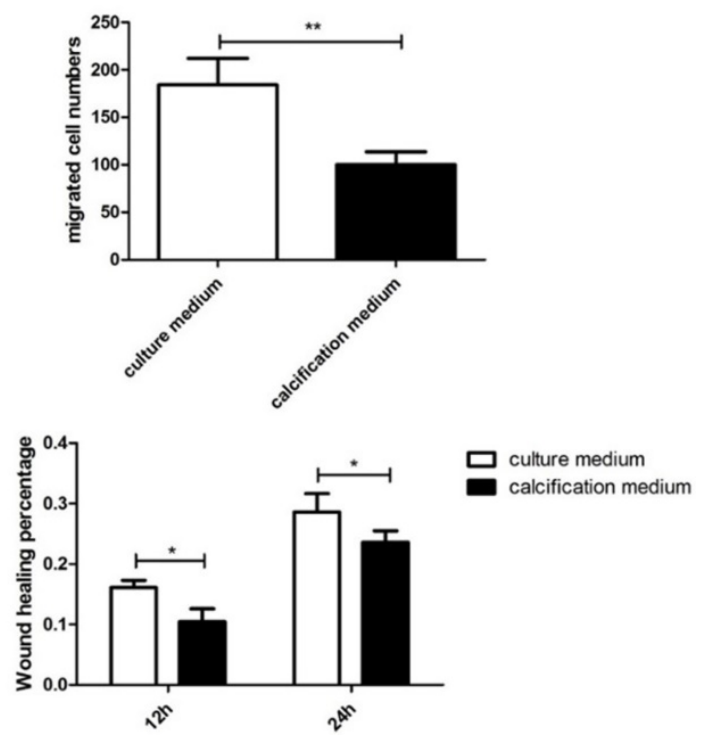

Figure 4. SKOV3 cell migration decreased after cultured with calcification medium for 21 d. (A) Typical optical images of SKOV3 which crossed through the pores of Transwell chamber and stained by crystal violet, illustrating cell migration at $24 \mathrm{~h}$, bar $=100 \mu \mathrm{m}$. (B) Quantitative analysis of the migrated cell number by Transwell assay. ${ }^{* *}$, indicated significant difference at $\mathrm{P}<0.01$. (C) Typical optical images illustrating cell migration at 0,12 and $24 \mathrm{~h}$ by scratch injury wound, bar $=200 \mu \mathrm{m}$. (D) Quantitative analysis of the wound healing percentage at 12 and $24 \mathrm{~h}$ using scratch injury wound. *, indicated significant difference at $P<0.05$. 


\section{Calcification of serous ovarian carcinomas}

We also verified our hypothesis on specimens. Laminated calcifications (psammoma body) in hematoxylin and eosin stain were seen in all the 40 biopsy specimens of calcified group, but not in the matched ovarian cancer specimens (Figure 5), which verified the diagnosis of the pathologist. Additionally, characteristics of patients were shown in the Table 1. Among the 40 calcified specimens, 9 cases were grade I, 16 cases were grade II, and 15 cases were grade III. Among the 40 non-calcified specimens, 7 cases were grade I, 7 cases were grade II, and 26 cases were grade III. There was statistically significant difference of the histological grade between calcified group and non-calcified group $(\mathrm{P}<0.05)$.

Table 1. Characteristic of the specimens.

\begin{tabular}{llll}
\hline Characteristic & non-calcification group & calcification group & P value \\
\hline Age & $53.25 \pm 10.72$ & $49.08 \pm 12.21$ & $0.476^{\mathrm{a}}$ \\
mean & 3 & 5 & $0.886^{\mathrm{b}}$ \\
$<35$ & 26 & 25 & $0.886^{\mathrm{b}}$ \\
$35-59$ & 11 & 10 & $0.886^{\mathrm{b}}$ \\
$>59$ & & & \\
Pathological grade & & 9 & $0.035^{\mathrm{c}}$ \\
Low & 7 & 16 & $0.035^{\mathrm{c}}$ \\
Medium & 7 & 15 & $0.035^{\mathrm{c}}$ \\
High & 26 & & \\
TNM stage & & 3 & $0.239 \mathrm{~b}$ \\
I & 6 & 11 & $0.239 \mathrm{~b}$ \\
II & 4 & 17 & $0.239 \mathrm{~b}$ \\
III & 18 & 9 & $0.239 \mathrm{~b}$ \\
IV & 10 & & \\
Lymph node & & 6 & $0.556^{\mathrm{c}}$ \\
metastasis & & 34 & $0.556^{\mathrm{c}}$ \\
\hline Yes & 8 & & \\
No & 32 & & \\
\hline a Two independent sample t test. & & \\
b Fisher's Exact Test. & & & \\
c Pearson Chi-Square. & & &
\end{tabular}

\section{Expression of mineralization biomarkers in serous ovarian carcinomas}

Expression of Collagen I, Runx2, $\beta 2$-microglobulin, BMP2, Osterix and OPN in serous ovarian carcinomas were shown in Figure 6 and scores of these mineralization biomarkers were present in Table 2. For the expression of the BMP2 and OPN, there was a statistically significant difference between calcified group and non-calcified group $(\mathrm{P}<0.05)$. But for the expression of the Collagen I, $\beta 2$-microglobulin, Runx2 and Osterix, there was no statistically significant difference. Although there was no statistically significant difference, the positive rate of Runx 2 and Osterix in calcified group was higher than the non-calcified group.
Table 2. Expression of Collagen I, Runx2, $\beta 2$-microglobulin, BMP2, Osterix and OPN with serous ovarian carcinomas

\begin{tabular}{|c|c|c|c|}
\hline & Non-calcified group & Calcified group & Pvalue \\
\hline \multicolumn{4}{|c|}{ Collagen I score } \\
\hline 0 & $1(2.5 \%)$ & $3(7.5 \%)$ & $0.227 \mathrm{a}$ \\
\hline 1 & $12(30 \%)$ & $6(15 \%)$ & $0.227 \mathrm{a}$ \\
\hline 2 & $27(70 \%)$ & $31(77.5 \%)$ & $0.227^{a}$ \\
\hline \multicolumn{4}{|c|}{ Runx2 score } \\
\hline 0 & $29(72.5 \%)$ & $22(55 \%)$ & $0.144^{a}$ \\
\hline 1 & $11(27.5 \%)$ & $16(40 \%)$ & $0.144^{a}$ \\
\hline 2 & $0(0 \%)$ & $2(5 \%)$ & $0.144^{a}$ \\
\hline \multicolumn{4}{|c|}{$\beta 2$-microglobulin score } \\
\hline 0 & $0(0 \%)$ & $2(5 \%)$ & $0.159 a$ \\
\hline 1 & $6(15 \%)$ & $2(5 \%)$ & $0.159 a$ \\
\hline 2 & $34(85 \%)$ & $36(90 \%)$ & $0.159^{a}$ \\
\hline \multicolumn{4}{|c|}{ BMP2 score } \\
\hline 0 & $26(65 \%)$ & $15(37.5 \%)$ & $0.042^{\mathrm{a}}$ \\
\hline 1 & $11(27.5 \%)$ & $22(55 \%)$ & $0.042^{\mathrm{a}}$ \\
\hline 2 & $3(7.5 \%)$ & $3(7.5 \%)$ & $0.042^{\mathrm{a}}$ \\
\hline \multicolumn{4}{|c|}{ Osterix score } \\
\hline 0 & $19(47.5 \%)$ & $16(40 \%)$ & $0.652^{\mathrm{a}}$ \\
\hline 1 & $21(52.5 \%)$ & $24(60 \%)$ & $0.652^{\mathrm{a}}$ \\
\hline 2 & $0(0 \%)$ & $0(0 \%)$ & $0.652^{\mathrm{a}}$ \\
\hline \multicolumn{4}{|c|}{ OPN score } \\
\hline 0 & $15(37.5 \%)$ & $3(7.5 \%)$ & $<0.0001^{\mathrm{b}}$ \\
\hline 1 & $20(50 \%)$ & $16(40 \%)$ & $<0.0001^{\mathrm{b}}$ \\
\hline 2 & $5(12.5 \%)$ & $21(52.5 \%)$ & $<0.0001^{\mathrm{b}}$ \\
\hline
\end{tabular}

\section{Discussion}

Calcification has a crucial role in ovarian cancer diagnosis and treatment. However, despite its value for the diagnosis of ovarian cancer and potential prognostic importance, the formation of calcification in ovarian cancer is unclear now. It remains uncertain whether it is caused by an active cellular process or it is caused by cellular degeneration. In this paper, we investigated ovarian calcification and hypothesized that the calcification could result from a mineralization process similar to that of osteogenesis. In this process, ovarian cancer cells switch to calcifying cells when induced by microenvironmental stimulatory factors.

First, calcification medium $(50 \mu \mathrm{g} / \mathrm{ml}$ vitamin C, $10 \mathrm{mM}$ beta glycerin sodium phosphate $+10^{-7} \mathrm{M}$ dexamethasone $+10 \%$ FBS) was used to establish an in vitro model of ovarian cancer calcification. Among them, the addition of exogenous phosphate is routine in mineralization studies of many pathologies, including osteoblasts, vascular smooth muscle cells and tumor cells in vitro[10], because the phosphate can participate in the formation of calcification such as hydroxyapatite. Dexamethasone, a synthetic glucocorticoid, can enhance the mineralization of certain bone-like cells[11]. Vitamin C, an essential co-factor for extracellular matrix synthesis in culture, since it is required for collagen synthesis, it has a marked effect on mineralization in vitro[12]. 

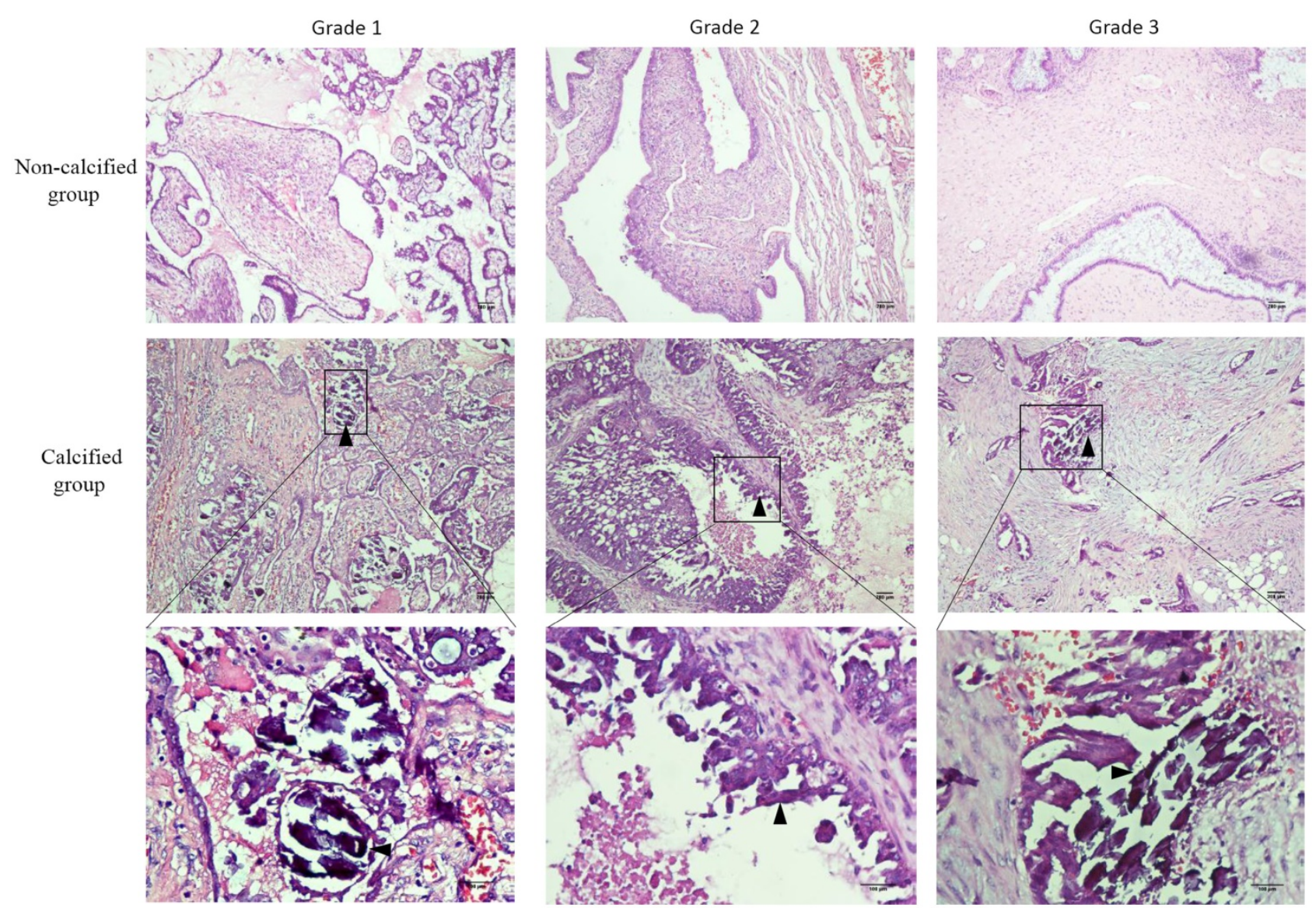

Figure 5. Calcification of serous ovarian carcinomas. $\Delta$ concentric, laminated calcifications (psammoma body) located in the stroma. The background

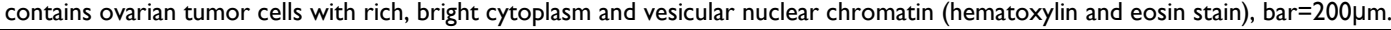

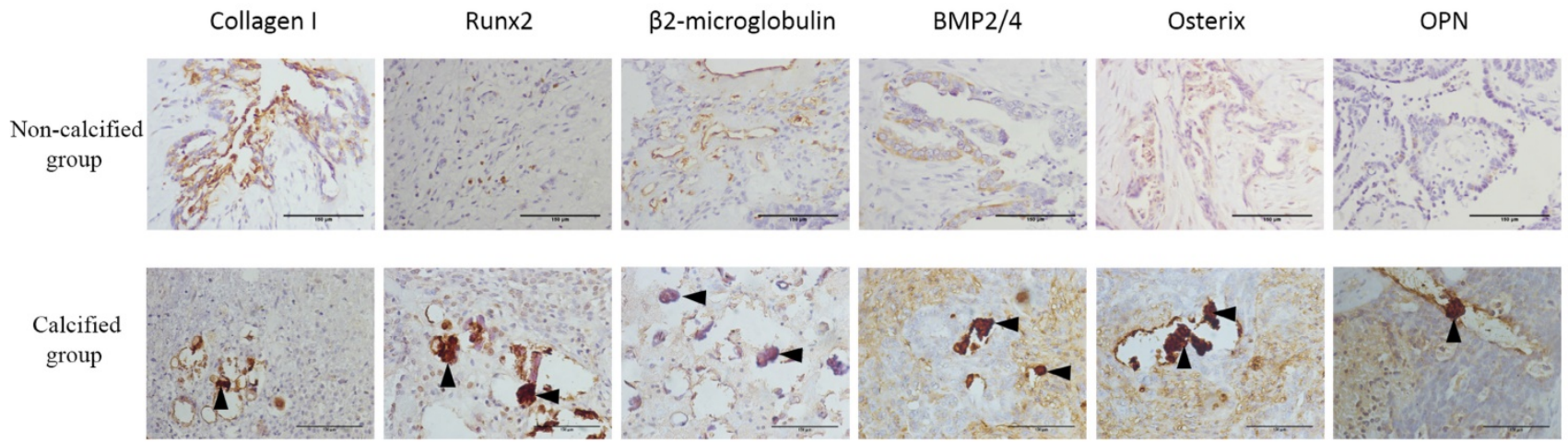

Figure 6. Expression of (A) Collagen I, (B) B2-microglobulin, (C) BMP2, (D) OPN, (E) Osterix, and (F) Runx2 (molecular markers of calcification) in serous ovarian carcinomas. $\triangle$, psammoma body, bar $=150 \mu \mathrm{m}$.

To examine whether mineralizing of ovarian cancer cell can be induced by calcification medium, we used several classical calcium salt detection methods (Alizarin red staining, von kossa staining, calcein fluorescence staining, ALP activity detection) to analyze the in vitro models of ovarian cancer calcification. Our results revealed that there were calcium deposits in the SKOV3 cells cultured with calcification medium compared with the SKOV3 cells cultured with culture medium or HOSEpiC cells, which suggested that SKOV3 cells had certain differentiation potential compared with the ovarian epithelial cells and could be induced differentiation by calcification medium. Thus, we have demonstrated here for the first time that ovarian cancer cell line SKOV3 is capable of mineralising in vitro and it can be induced mineralizing by calcification medium.

Second, we used the established in vitro model of ovarian cancer calcification to investigate the changes of ultrastructure and the mineralization biomarkers 
after the induction of calcification medium. Under transmission electron microscope (TEM), terminal differentiation occurred in SKOV3 cells cultured with calcification medium, which was similar to the osteoblast phenotype. What's more, the expression of mineralization biomarkers (CollagenI, Runx2, 32-microglobulin, BMP2, Osterix and OPN) was elevated in calcifying SKOV3 cells, which suggested that calcifying SKOV3 cells may maintain an calcifying phenotype. From above, the in vitro study fully demonstrated that after cultured under calcification medium, ovarian cancer cells already obtained osteoblast-like characteristics.

Our study of specimens also demonstrated that mineralization biomarkers BMP2, OPN, Runx2 and Osterix were present more in the calcified serous ovarian carcinomas, and there was statistically significant difference of the expression of the BMP2 and OPN between calcified group and non-calcified group $(\mathrm{P}<0.05)$. These results imply a possible link between BMP2, OPN, Runx2, Osterix and calcified serous ovarian carcinomas. As a typical member of the BMP family, BMP2 has obvious osteoinductive activities, such as directly enhancing cell calcification and inducing new bone formation[13]. BMP2 modulates osteogenesis via the BMP2 pathway. Through the BMP/Smad pathways[14], signals from the pathways are transmitted to regulate the synthesis of osteogenic factors including OPN. The transcription factors such as Runx2 and Osterix can control downstream target genes including OPN to play an important role in the differentiation and maturation of osteoblasts and to regulate the osteoblast proliferation[15]. Osterix is generally thought to exist downstream of Runx2, and Runx2 plays a role in early osteogenic differentiation, and Osterix plays a role in the late stage of osteogenic differentiation[16]. The predominant occurrence of these mineralization biomarkers in the calcified serous ovarian carcinomas demonstrates an osteogenesis process in the calcified serous ovarian carcinomas. However, regarding the small amount of our study of specimens, the results should be explained with great caution, and more large-scale studies are needed.

When the SKOV3 cell switched its phenotype to osteoblast-like phenotype, they became welldifferentiated. Scratch wound assays and Transwell assay found that SKOV3 cell migration decreased after being cultured with calcification medium, which implied a well-differentiated phenotype. In our study of specimens, the psammoma bodies occurred predominantly in well-differentiated carcinomas, which is consistent with many other studies and suggested that the presence of calcification was correlated with a lower histologic grade. In the study by Burkill and colleagues, 78/107 (73\%) patients with calcification had stage I-II disease, whereas 511/1055 $(48 \%)$ patients without calcification had stage I-II disease[3]. Likewise, Silva revealed the same result[7]. The main presence of calcification in cases of low-grade carcinomas supports the theory that calcification in ovary cancer is rarely due to cellular degeneration, because calcification will occur more frequently in high-grade carcinomas if it is mainly caused by cellular degeneration. In light of our observation on the locations of calcification, we found that the calcification mainly located in the stroma rather than in the epithelium. The locations of calcification in the stroma also suggests that calcification is not associated with cellular degeneration. If calcification is mainly caused by cellular degeneration, then the epithelial cells with a higher proliferation rate will be more prone to degenerative changes and the locations of calcification will mainly be in the epithelium.

So how does the cancer cell switch its phenotype to osteoblast-like phenotype? Some studies reported that this phenomenon might related with EMT(epithelial mesenchymal transition). In breast cancer, the process of dedifferentiation of breast cancer cells could related to the EMT markers[17]. After the dedifferentiation mediated by the EMT, breast cancer cells may acquire mesenchymal characteristics and transform themselves into cells with an osteoblast-like phenotype[18]. In this transformation, the BMP2/RUNX2 signaling pathway were activated and the bone-related genes were ectopically co-expressed in breast cancer cells[19]. Another pathway that needs to pay attention to is RANK/RANKL signaling. Bone metastases in breast cancer are most often osteolytic and the generation of osteolytic metastases is the activation of osteoclasts[20]. Osteoblasts secrete RANKL, which results in the activation and finally maturation of osteoclasts[21]. It is interesting to note that RANKL also involved in breast cancer cells and seem to play a role in bone metastasis[20]. A process opposite to the EMT, MET(mesenchymal to epithelial transition), is an evolving and relatively under-investigated mechanism that is considered to contribute substantially to the colonisation of breast cancer cells into metastatic site[22]. When the breast cancer cells acquire the osteoblast-like phenotype, they also acquire the high affinity for bone environment, which may explain the high frequency in which breast cancer metastasize into bone[23]. These evidences highlight the role of EMT and MET in breast cancer osteoblast-like cells occurrence and cancer bone metastasis. 
As mentioned previously, the breast cancer cells obtaining an osteoblast-like feature via EMT may associated with the initiation of the metastatic cascade. And its reverse process, known as the MET, is necessary for efficient bone colonization. The ovarian cancer cells switch its phenotype to osteoblast-like phenotype may experience a similar process as the breast cancer do. However, considering the less incidence of bone metastases in ovarian cancer and the decreased migration after the osteogenic phenotype transformation, the EMT and MET process in the ovarian osteogenic phenotype transformation may have some difference from the breast osteogenic phenotype transformation. Further study on the EMT and MET process in the ovarian osteogenic phenotype transformation is needed.

It is important to note that EMT and MET is a balance process. EMT and MET may determine active or dormant states of the tumor, respectively, and allow for an indeterminate number of cycles of invasion and metastases formation[22]. MET, reverting the mesenchymal cells back to epithelial cells, may reduce the EMT-associated malignancy [24]. Additionally, although MET is the driving force of metastatic colonization, tumors that have undergone a MET at a secondary site may be less invasive and more susceptible to chemotherapeutic drugs [25]. If we could interfere the EMT and MET process in the ovarian osteogenic phenotype transformation to reduce EMT and promote MET, we can promote the terminal differentiation of tumor cells in the primary site and inhibit the spread of ovarian cancer cells. Thus, experimental studies will be needed to settle the key question whether inducing or inhibiting MET would be beneficial in the treatment of ovarian cancer. Further, making clear the pathways that contribute the MET in the ovarian osteogenic phenotype transformation is also important. Understanding the precise mechanisms of EMT and MET in the ovarian osteogenic phenotype transformation can inform the new prospective in ovarian cancer therapy.

Based on the results discussed in this paper, we propose a mechanism for the calcification in ovarian cancer (Figure 7). We suggest that ovarian cancer cells switch to a calcifying phenotype and express bone related transcription factors and bone matrix proteins to cause the calcification deposition. Additionally, understanding the formation of calcifications in ovarian cancer might have a positive influence on the development of new diagnostic and therapeutic modalities for the ovarian cancer.

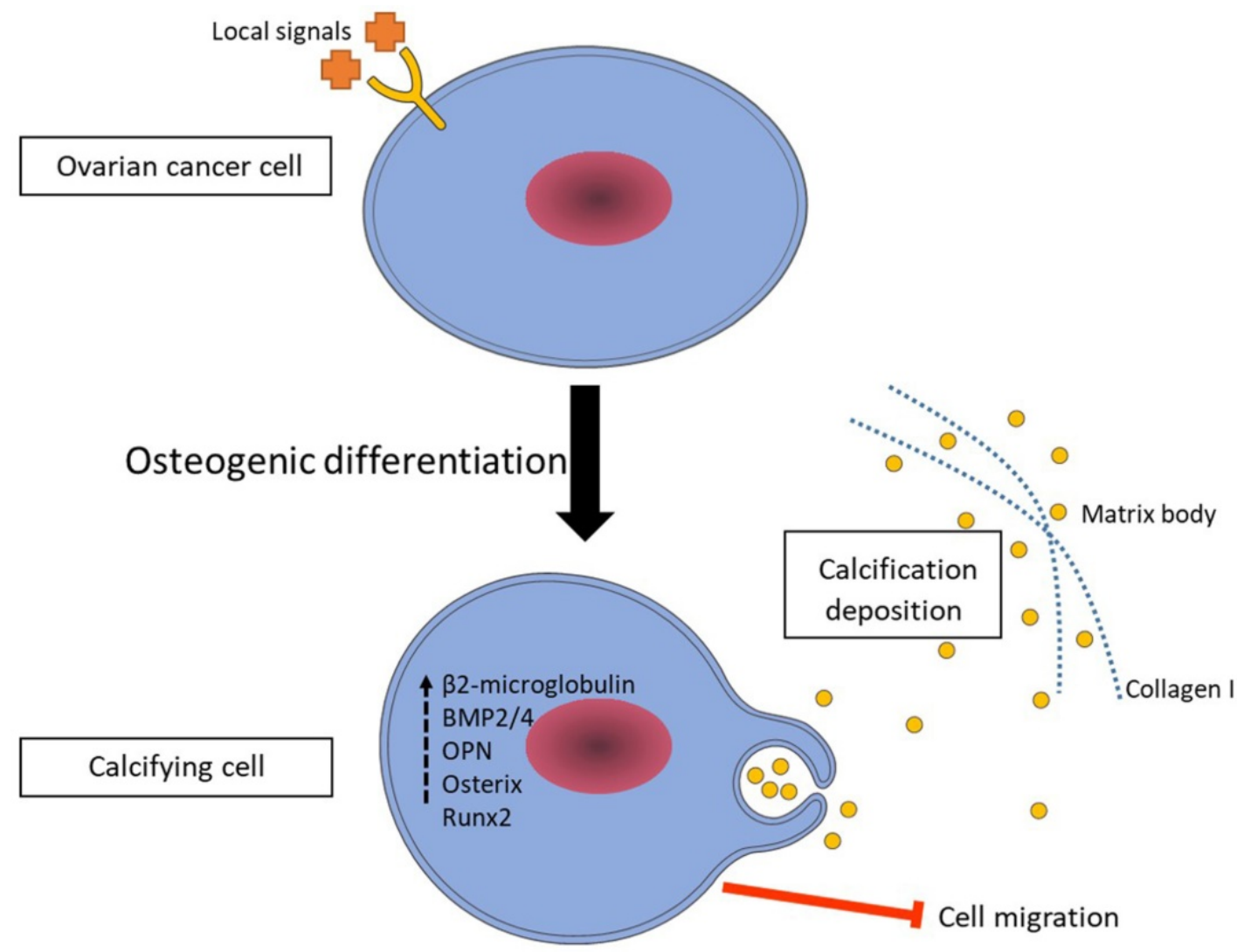

Figure 7. A proposed mechanism of ovarian cancer cell calcification. According to our results, ovarian cancer cells switch to a calcifying phenotype and express bone related transcription factors and bone matrix proteins to cause the calcification deposition. 


\section{Acknoledgements}

1. National Natural Science Foundation of China (Grant No. 11262020) 2. Foundation of Sichuan Provincial Science and Technology Program (Grant No. 2016SZ0020, 2014FZ0074, 2014SZ0002-15).

\section{Competing Interests}

The authors have declared that no competing interest exists.

\section{References}

1. Siegel RL, Miller KD, Jemal A. Cancer Statistics, 2017. Ca-Cancer J Clin. 2017; 67: 7-30.

2. Naora H, Montell DJ. Ovarian cancer metastasis: integrating insights from disparate model organisms. Nat Rev Cancer. 2005; 5: 355-66.

3. Burkill GJ, Allen SD, A'Hern R P, Gore ME, King DM. Significance of tumour calcification in ovarian carcinoma. Br J Radiol. 2009; 82: 640-4.

4. Das DK. Psammoma Body: A Product of Dystrophic Calcification or of a Biologically Active Process That Aims at Limiting the Growth and Spread of Tumor? Diagn Cytopathol. 2009; 37: 534-41.

5. Friesen MA, White SV, Byers JF. Handoffs: Implications for Nurses. In: Hughes RG, editor. Patient Safety and Quality: An Evidence-Based Handbook for Nurses. Rockville (MD); 2008.

6. Cox RF, Hernandez-Santana A, Ramdass S, McMahon G, Harmey JH, Morgan MP. Microcalcifications in breast cancer: novel insights into the molecular mechanism and functional consequence of mammary mineralisation. Br J Cancer. 2012; 106: 525-37.

7. Silva EG, Deavers MT, Parlow AF, Gershenson DM, Malpica A. Calcifications in ovary and endometrium and their neoplasms. Mod Pathol. 2003; 16: 219-22.

8. Feng W, Zhang K, Liu Y, Chen J, Cai Q, Zhang Y, et al. Apocynin attenuates angiotensin II-induced vascular smooth muscle cells osteogenic switching via suppressing extracellular signal-regulated kinase 1/2. Oncotarget. 2016; 7: 83588-600.

9. Scimeca M, Giannini E, Antonacci C, Pistolese CA, Spagnoli LG, Bonanno E. Microcalcifications in breast cancer: an active phenomenon mediated by epithelial cells with mesenchymal characteristics. Bmc Cancer. 2014; 14.

10. Sun Y, Byon CH, Yuan K, Chen J, Mao X, Heath JM, et al. Smooth muscle cell-specific runx2 deficiency inhibits vascular calcification. Circ Res. 2012; 111: 543-52.

11. Zhan JK, Wang YJ, Wang Y, Tang ZY, Tan P, Huang W, et al. The protective effect of GLP-1 analogue in arterial calcification through attenuating osteoblastic differentiation of human VSMCs. Int J Cardiol. 2015; 189: 188-93.

12. Boskey AL, Stiner D, Doty SB, Binderman I. Requirement of vitamin C for cartilage calcification in a differentiating chick limb-bud mesenchymal cell culture. Bone. 1991; 12: 277-82.

13. Shioi A, Nishizawa $\mathrm{Y}$, Jono S, Koyama H, Hosoi M, Morii $\mathrm{H}$ Beta-glycerophosphate accelerates calcification in cultured bovine vascular smooth muscle cells. Arterioscler Thromb Vasc Biol. 1995; 15: 2003-9.

14. Chen D, Zhao M, Harris SE, Mi Z. Signal transduction and biological functions of bone morphogenetic proteins. Frontiers in bioscience : a journal and virtual library. 2004; 9: 349-58

15. Bruderer $M$, Richards RG, Alini M, Stoddart MJ. Role and regulation of RUNX2 in osteogenesis. Eur Cell Mater. 2014; 28: 269-86.

16. Nakashima K, Zhou X, Kunkel G, Zhang ZP, Deng JM, Behringer RR, et al. The novel zinc finger-containing transcription factor Osterix is required for osteoblast differentiation and bone formation. Cell. 2002; 108: 17-29.

17. Scimeca M, Antonacci C, Colombo D, Bonfiglio R, Buonomo OC, Bonanno E. Emerging prognostic markers related to mesenchymal characteristics of poorly differentiated breast cancers. Tumour biology : the journal of the International Society for Oncodevelopmental Biology and Medicine. 2016; 37: 5427-35.

18. Scimeca M, Chiara A, Bonanno E. Breast Microcalcifications: A Focus. Journal of cell science. 2015; s8.

19. Tan CC, Li GX, Tan LD, Du X, Li XQ, He R, et al. Breast cancer cells obtain an osteomimetic featureviaepithelial-mesenchymal transition that have undergone BMP2/RUNX2 signaling pathway induction. Oncotarget. 2016; 7: 79688-705.

20. Weidle UH, Birzele F, Kollmorgen G, Ruger R. Molecular Mechanisms of Bone Metastasis. Cancer genomics \& proteomics. 2016; 13: 1-12

21. Dougall WC. Molecular pathways: osteoclast-dependent and osteoclast-independent roles of the RANKL/RANK/OPG pathway in tumorigenesis and metastasis. Clinical cancer research : an official journal of the American Association for Cancer Research. 2012; 18: 326-35.

22. Demirkan B. The Roles of Epithelial-to-Mesenchymal Transition (EMT) and Mesenchymal-to-Epithelial Transition (MET) in Breast Cancer Bone Metastasis: Potential Targets for Prevention and Treatment. J Clin Med. 2013; 2. 264-82.
23. Scimeca M, Starace F, Gratti D, Sapia V, Bonfiglio R, Bonanno E. Osteotropism of Breast and Prostate Cancer: Clinical and Experimental Perspective. Int J Immunother Cancer Res. 2017; 3: 032-3.

24. Zhu LY, Zhang WM, Yang XM, Cui L, Li J, Zhang YL, et al. Silencing of MICAL-L2 suppresses malignancy of ovarian cancer by inducing mesenchymal-epithelial transition. Cancer Lett. 2015; 363: 71-82.

25. Tseng JH, Bisogna $\mathrm{M}$, Hoang LN, Olvera N, Rodriguez-Aguayo $C_{\text {, }}$ Lopez-Berestein G, et al. miR-200c-driven Mesenchymal-To-Epithelial Transition is a Therapeutic Target in Uterine Carcinosarcomas. Sci Rep. 2017; $7: 3614$ 